\title{
Scalar-isovector $\delta$-meson mean-field and mixed phase structure in compact stars
}

\author{
Grigor Bakhshi Alaverdyan
}

Department of Radio Physics, Yerevan State University, Yerevan, Armenia; galaverdyan@ysu.am

Received 16 December 2009; revised 6 March 2010; accepted 15 March 2010.

\begin{abstract}
The deconfinement phase transition from hadronic matter to quark matter in the interior of compact stars is investigated. The hadronic phase is described in the framework of relativistic mean-field (RMF) theory, when also the scalar-isovector $\delta$-meson effective field is taken into account. The MIT bag model for describing a quark phase is used. The changes of the parameters of phase transition caused by the presence of $\delta$-meson field are investigated. Finally, alterations in the integral and structure parameters of hybrid stars due to deconfinement phase transitions are discussed.
\end{abstract}

Keywords: Neutron Stars; Equation of State; Relativistic Mean-Field; Quarks; Deconfinement Phase Transition

\section{INTRODUCTION}

Study of the structure characteristics and composition of the matter constituents at extremely high density region is of great interest in both nuclear and neutron star physics. The RMF theory [1] has been effectively applied to describe the structure of finite nuclei, the features of heavy-ion collisions, and the equation of state (EOS) of nuclear matter. Inclusion of the scalar-isovector $\delta$ meson in this scheme and investigation of its influence on low density asymmetric nuclear matter was realized in $[2,3]$. At sufficiently high density, different exotic degrees of freedom, such as pion and kaon condensates, also deconfined quarks, may appear in the strongly interacting matter. The modern concept of hadron-quark phase transition is based on the feature of that transition, that is the presence of two conserved quantities in this transition: baryon number and electric charge [4]. It is known that, depending on the value of surface tension, $\sigma_{\mathrm{s}}$, the phase transition of nuclear matter into quark matter can occur in two scenarios [5,6]: ordinary first order phase transition with a density jump (Maxwell construction), or formation of a mixed hadron-quark matter with a continuous variation of pressure and density (Glendenning construction) [4]. Uncertainty of the surface tension values does not allow to determine the phase transition scenario, taking place in realty. In our recent paper [7] in the assumption that the transition to quark matter is a usual first-order phase transition, described by Maxwell construction, we have shown that the presence of the $\delta$-meson field leads to the decrease of transition pressure $P_{0}$, of baryon number densities $n_{N}$ and $n_{Q}$.

In this article we investigate the hadron-quark phase transition of neutron star matter, when the transition proceeds through a mixed phase. Influence of $\delta$-meson field on such phase transition characteristics and of compact star structure is discussed.

\section{NEUTRON STAR MATTER EOS WITH DECONFINEMENT PHASE TRANSITION}

\subsection{Nuclear Matter}

For description of hadronic phase we use the relativistic Lagrangian density of many-particle system consisting of nucleons, p, n, and exchanged mesons $\sigma, \omega, \rho, \delta$ :

$$
\begin{gathered}
\mathrm{L}_{\sigma \omega \rho \delta}\left(\sigma(x), \omega_{\mu}(x), \vec{\rho}_{\mu}(x), \vec{\delta}(x)\right) \\
=\mathrm{L}_{\sigma \omega \rho}\left(\sigma(x), \omega_{\mu}(x), \vec{\rho}_{\mu}(x)\right) \\
+U(\sigma(x))+\mathrm{L}_{\delta}(\vec{\delta}(x)),
\end{gathered}
$$

where

$\mathrm{L}_{\sigma \omega \rho}\left(\sigma(x), \omega_{\mu}(x), \vec{\rho}_{\mu}(x)\right)$ is the linear part of relativistic Lagrangian density without $\delta$-meson field [8], $U(\sigma)$ $=\frac{b}{3} m_{N}\left(g_{\sigma} \sigma\right)^{3}+\frac{c}{4}\left(g_{\sigma} \sigma\right)^{4} \quad$ and $\quad \mathrm{L}_{\delta}(\vec{\delta})=\frac{1}{2}\left(\partial_{\mu} \vec{\delta} \partial^{\mu} \vec{\delta}\right.$ $-m_{\delta} \vec{\delta}^{2}$ ) are the $\sigma$-meson self-interaction term and 
contribution of the $\delta$-meson field, respectively. This Lagrangian density (1) contains the meson-nucleon coupling constants, $g_{\sigma}, g_{\omega}, g_{\rho}, g_{\delta}$ and also parameters of $\sigma$-field self-interacting terms, $b$ and $c$. In our calculations we take $a_{\delta}=\left(g_{\delta} / m_{\delta}\right)^{2}=2.5 \mathrm{fm}^{2}$ for the $\delta$ coupling constant, as in [2,7]. Also we use $m_{N}=938.93 \mathrm{MeV}$ for the bare nucleon mass, $m_{N}^{*}=0.78 m_{N}$ for the nucleon effective mass, $n_{0}=0.153 \mathrm{fm}^{-3}$ for the baryon number density at saturation, $f_{0}=-16.3 \mathrm{MeV}$ for the binding energy per baryon, $K=300 \mathrm{MeV}$ for the incompressibility modulus, and $E_{\text {sym }}^{(0)}=32.5 \mathrm{MeV}$ for the asymmetry energy. Five other constants, $a_{\sigma}=\left(g_{\sigma} / m_{\sigma}\right)^{2}, a_{\omega}=\left(g_{\omega} / m_{\omega}\right)^{2}$, $a_{\rho}=\left(g_{\rho} / m_{\rho}\right)^{2}, b$ and $c$, then can be numerically determined: $a_{\sigma}=9.154 \mathrm{fm}^{2}, \quad a_{\omega}=4.828 \mathrm{fm}^{2}, \quad a_{\rho}=$ $13.621 \mathrm{fm}^{2}, b=0.01654 \mathrm{fm}^{-1}, c=0.01319$. When we neglect the $\delta$ channel, then $a_{\delta}=0$ and $a_{\rho}=$ $4.794 \mathrm{fm}^{2}$. The knowledge of the model parameters makes it possible to solve the set of four equations in a self-consistent way and to determine the re-denoted mean-fields, $\sigma \equiv g_{\sigma} \bar{\sigma}, \omega \equiv g_{\omega} \overline{\omega_{0}}, \rho \equiv g_{\rho} \overline{\rho_{0}^{(3)}}$, and $\delta \equiv g_{\delta} \overline{\delta^{(3)}}$ depending on baryon number density $n$ and asymmetry parameter $\alpha=\left(n_{n}-n_{p}\right) / n$. The standard QHD procedure allows to obtain expressions for energy density $\varepsilon_{N M}(n, \alpha)$ and pressure $P_{N M}(n, \alpha)$ [7]. In Figure 1 we illustrate the 3D-plot of the energy per baryon, $E_{b}(n, \alpha)=\varepsilon_{N M}(n, \alpha) / n$ as a function of the baryon number density $n$ and asymmetry parameter $\alpha$ in case of $\beta$-equilibrium charged npe-plasma. The curves correspond to different fixed values of the charge per baryon, $q=\left(n_{p}-n_{e}\right) / n$. The thick one corresponds to $\beta$-equilibrium charge neutral npe-matter. The lower and upper surfaces corresponds to $R M F_{\sigma \omega \rho}$ and $R M F_{\sigma \omega \rho \delta}$ models respectively.

Clearly, including a $\delta$-meson field increases the energy per baryon, and this change is greater for larger values of the asymmetry parameter. For a fixed value of the specific charge, the asymmetry parameter falls off monotonically as the density is increased.

In Figure 2 we plotted the effective mean-fields of exchanged mesons, $\sigma, \omega, \rho$ and $\delta$ as a function of the baryon number density $n$ for the charge-neutral $\beta$-equilibrium npe-plasma. The solid and dashed lines correspond to the $R M F_{\sigma \omega \rho \delta}$ and $R M F_{\sigma \omega \rho}$ models, respectively.
From Figure 2 one can see that the inclusion of the scalar-isovector virtual $\delta\left(a_{0}(980)\right)$ meson results in significant changes of the $\rho$ and $\delta$ meson effective fields. This can result in changes of deconfinement

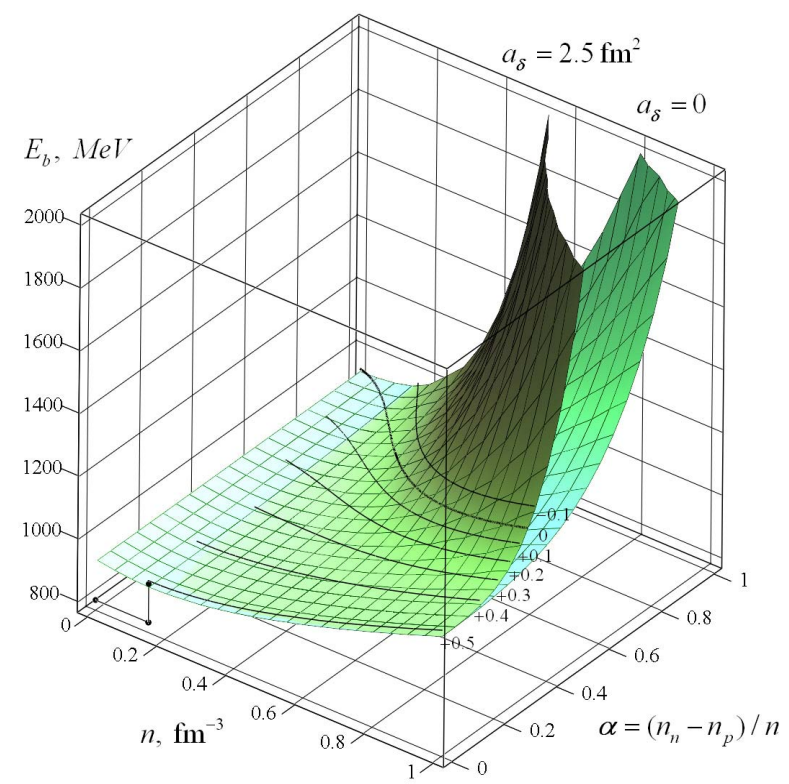

Figure 1. Energy per baryon $E_{b}$ as a function of the baryon number density $n$ and the asymmetry parameter $\alpha$ in case of a $\beta$-equilibrium charged npe-plasma.

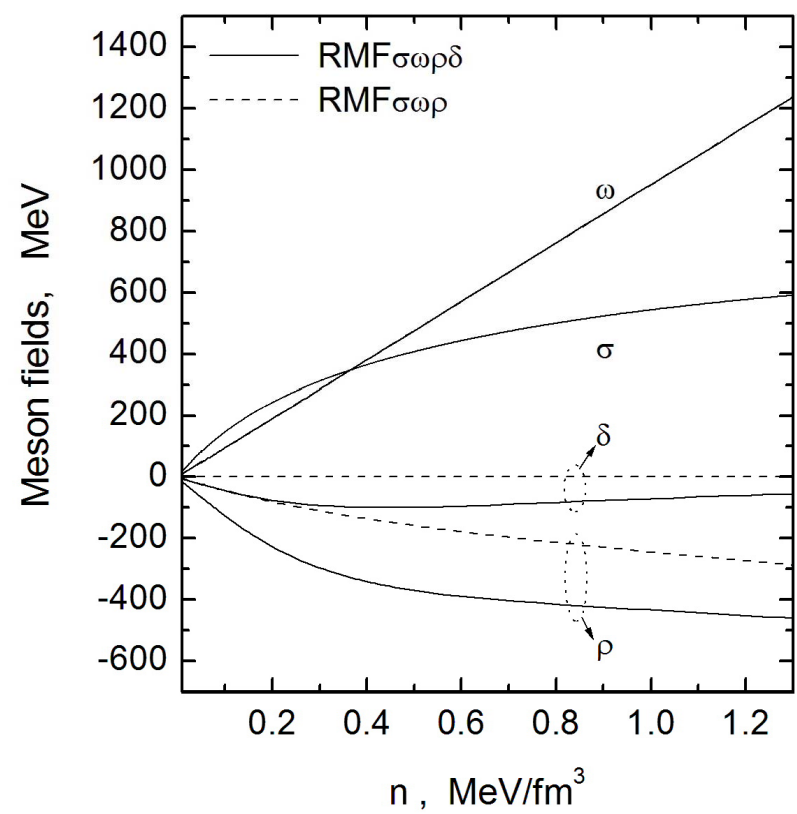

Figure 2. Re-denoted meson mean-fields as a function of the baryon number density $n$ in case of a $\beta$-equilibrium chargeneutral npe-plasma with and without $\delta$-field. 
phase transition parameters and, thus, alter the structural characteristics of neutron stars. The results of our analysis show that the scalar - isovector $\delta$-meson field inclusion leads to the increase of the EOS stiffness of nuclear matter due to the splitting of proton and neutron effective masses, and also due to the increase of asymmetry energy (for details see Ref. [9]).

\subsection{Quark Matter}

To describe the quark phase an improved version of the MIT bag model is used, in which the interactions between $u, d$ and $s$ quarks inside the bag are taken into account in the one-gluon exchange approximation [10]. We choose $m_{u}=5 \mathrm{MeV}, m_{d}=7 \mathrm{MeV}$ and $m_{s}=150$ $\mathrm{MeV}$ for quark masses, $B=60 \mathrm{MeV} / \mathrm{fm}^{3}$ for bag parameter and $\alpha_{s}=0.5$ for the strong interaction constant.

\subsection{Deconfinement Phase Transition Parameters}

There are two independent conserved charges in hadron quark phase transition: baryonic charge and electric charge. The constituents chemical potentials of the npe-plasma in $\beta$-equilibrium are expressed through two potentials, $\mu_{b}$ and $\mu$, according to conserved charges. The pressure $P_{N M}$, energy density $\varepsilon_{N M}$ and baryon number density $n_{N M}$, are functions of independent potentials, $\mu_{b}^{(N M)}$ and $\mu_{e l}^{(N M)}$.

The thermodynamic characteristics, pressure $P_{Q M}$, energy density $\varepsilon_{Q M}$ and baryon number density $n_{Q M}$, are functions of chemical potentials $\mu_{b}^{(Q M)}$ and $\mu_{e l}^{(Q M)}$.

The mechanical and chemical equilibrium conditions (Gibbs conditions) for mixed phase are

$$
\begin{gathered}
\mu_{b}^{(Q M)}=\mu_{b}^{(N M)}=\mu_{b}, \quad \mu_{e l}^{(Q M)}=\mu_{e l}^{(N M)}=\mu_{e l}, \\
P_{Q M}\left(\mu_{b}, \mu_{e l}\right)=P_{N M}\left(\mu_{b}, \mu_{e l}\right) .
\end{gathered}
$$

The volume fraction of quark phase is

$$
\chi=V_{Q M} /\left(V_{Q M}+V_{N M}\right)
$$

where $V_{Q M}$ and $V_{N M}$ are volumes occupied by quark matter and nucleonic matter, respectively.

We applied the global electrical neutrality condition for mixed quark-nucleonic matter, according to Glendenning $[4,8]$,

$$
\begin{array}{r}
\frac{1}{3} \chi\left(2 n_{u}\left(\mu_{b}, \mu_{e l}\right)-n_{d}\left(\mu_{b}, \mu_{e l}\right)-n_{s}\left(\mu_{b}, \mu_{e l}\right)\right) \\
+(1-\chi) n_{p}\left(\mu_{b}, \mu_{e l}\right)-n_{e}\left(\mu_{e l}\right)=0 .
\end{array}
$$

The baryon number density in the mixed phase is determined as

$$
\begin{array}{r}
n=\frac{1}{3} \chi\left(n_{u}\left(\mu_{b}, \mu_{e l}\right)+n_{d}\left(\mu_{b}, \mu_{e l}\right)+n_{s}\left(\mu_{b}, \mu_{e l}\right)\right) \\
+(1-\chi)\left(n_{p}\left(\mu_{b}, \mu_{e l}\right)+n_{n}\left(\mu_{b}, \mu_{e l}\right)\right)
\end{array}
$$

and the energy density is

$$
\begin{aligned}
\varepsilon= & \chi\left(\varepsilon_{u}\left(\mu_{b}, \mu_{e l}\right)+\varepsilon_{d}\left(\mu_{b}, \mu_{e l}\right)+\varepsilon_{s}\left(\mu_{b}, \mu_{e l}\right)\right) \\
& +(1-\chi)\left(\varepsilon_{p}\left(\mu_{b}, \mu_{e l}\right)+\varepsilon_{n}\left(\mu_{b}, \mu_{e l}\right)\right)+\varepsilon_{e}\left(\mu_{e l}\right) .
\end{aligned}
$$

In case of $\chi=0$, the chemical potentials $\mu_{b}^{N}$ and $\mu_{e l}^{N}$, corresponding to the lower threshold of a mixed phase, are determined by solving Eq.3 and Eq.5. This allows to find the lower boundary parameters $P_{N}, \varepsilon_{N}$ and $n_{N}$. Similarly, we calculate the upper boundary values of mixed phase parameters, $P_{Q}, \varepsilon_{Q}$ and $n_{Q}$, corresponding to $\chi=1$.

The system of Eqs.3, 5, 6 and 7 makes it possible to determine EOS of mixed phase between these critical states.

Note, that in the case of an ordinary first-order phase transition both nuclear and quark matter are assumed to be separately electrically neutral, and at some pressure $P_{0}$, corresponding to the coexistence of the two phases, their baryon chemical potentials are equal, i.e.,

$$
\mu_{N M}\left(P_{0}\right)=\mu_{Q M}\left(P_{0}\right)
$$

Such phase transition scenario is known as phase transition with constant pressure (Maxwell construction).

Table 1 represents the parameter sets of the mixed phase both with and without $\delta$-meson field. It is shown that the presence of $\delta$-field alters threshold characteristics of the mixed phase. The lower threshold parameters, $n_{N}, \varepsilon_{N}, P_{N}$ are increased, meanwhile the upper ones $n_{Q}, \varepsilon_{Q}, P_{Q}$ are slowly decreased.

In Figure 3 we plot the species number densities as a function of baryon density $n$ for Glendenning construction. Quarks appear at the critical density $n_{N}=0.077 \mathrm{fm}^{-3}$.

Table 1. The mixed phase parameters with and without $\delta$ meson field.

\begin{tabular}{lll}
\hline Model & $R M F_{\sigma \omega \rho}$ & $R M F_{\sigma \omega \rho \delta}$ \\
\hline$n_{N}, \mathrm{fm}^{-3}$ & 0.0717 & 0.0771 \\
$n_{Q}, \mathrm{fm}^{-3}$ & 1.0830 & 1.0830 \\
$\varepsilon_{N}, \mathrm{MeV} / \mathrm{fm}^{3}$ & 67.728 & 72.793 \\
$\varepsilon_{Q}, \mathrm{MeV} / \mathrm{fm}^{3}$ & 1280.889 & 1280.884 \\
$P_{N}, \mathrm{MeV} / \mathrm{fm}^{3}$ & 0.336 & 0.434 \\
$P_{Q}, \mathrm{MeV} / \mathrm{fm}^{3}$ & 327.747 & 327.745 \\
\hline
\end{tabular}


The hadronic matter completely disappears at $n_{Q}=1.083$ $\mathrm{fm}^{-3}$, where the pure quark phase occurs.

Using the obtained EOS of nuclear matter, we have integrated the Tolman-Oppenheimer Volkoff equations and obtained the mass $M$ and the radius $R$ of compact stars for the different values of central pressure $P_{c}$.

Figure 4 illustrate the $M(R)$ dependence of neutron stars. We can see, that the behavior of mass-radius dependence significantly differs for the two types of phase transitions. Figure 4 shows that for $B=60 \mathrm{MeV} / \mathrm{fm}^{3}$ there are unstable regions, where $d M / d P_{c}<0$ between two stable branches of compact stars, corresponding to configurations with and without quark matter. In this case, there is a nonzero minimum value of the quark phase core radius. Accretion of matter on a critical neutron star configuration will then result in a catastrophic rearrangement of the star, forming a star with a quark matter core. The range of mass values for stars, containing the mixed phase, is $\left[0.085 M_{\odot} ; 1.853 M_{\odot}\right]$. In case of Maxwellian type phase transition, the analogous range is $\left[0.216 M_{\odot} ; 1.828 M_{\odot}\right]$.

\section{CONCLUSIONS}

In this paper we have studied the deconfinement phase transition of neutron star matter, when the nuclear matter is described in the RMF theory with $\delta$-meson effective field. We show that the inclusion of scalar - isovector $\delta$-meson field terms leads to the stiff nuclear matter

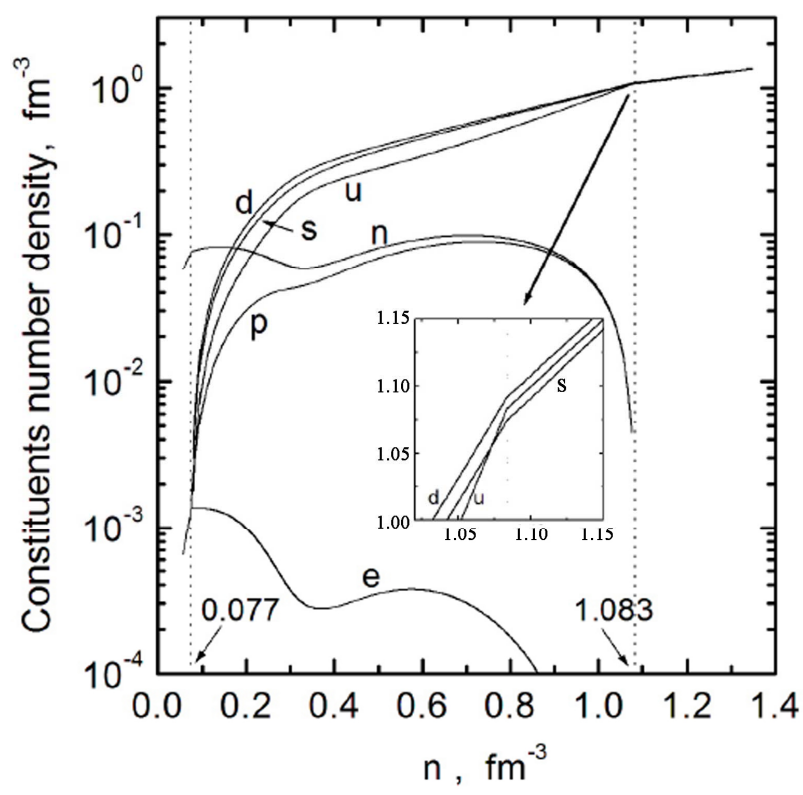

Figure 3. Constituents number density vs. baryon number density in case of Glendenning construction. Vertical dotted lines represent the mixed phase boundaries.

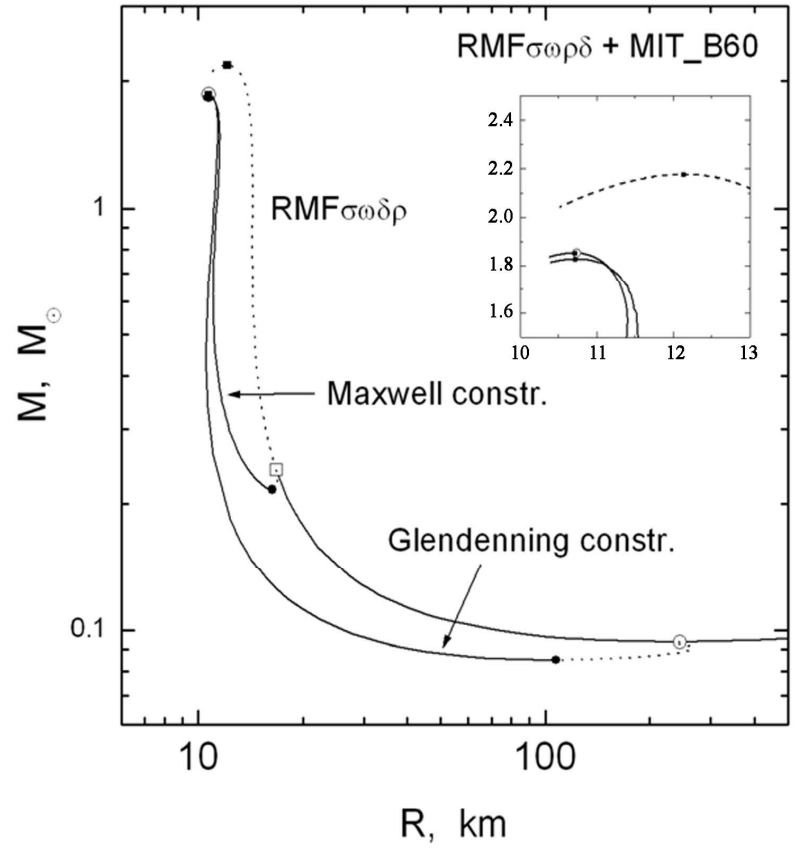

Figure 4. The mass-radius relation of neutron star with different deconfinement phase transition scenarios. Open circles and squares denote the critical configurations for Glendenning and Maxwellian type transitions, respectively. Solid circles and squares denote hybrid stars with minimal and maximal masses, respectively.

EOS. In a nucleonic star both the gravitational mass and corresponding radius of the maximum mass stable configuration increases with the inclusion of the $\delta$-field. The presence of scalar - isovector $\delta$-meson field alters the threshold characteristics of the mixed phase. The lower threshold parameters, $n_{N}, \varepsilon_{N}, P_{N}$ are increased, while the upper thresholds, $n_{Q}, \varepsilon_{Q}, P_{Q}$, are slowly decreased. For EOS used in this study, the central pressure of the maximum mass neutron stars is less than the mixed phase upper threshold $P_{Q}$. The maximum mass configuration has a gravitational mass $M_{\max }=1.853 M_{\odot}$ with radius $R_{m}=10.71 \mathrm{~km}$, and central density $\rho_{c}=2.32210^{15} \mathrm{~g} / \mathrm{cm}^{3}$. This star has a pure strange quark matter core with radius $r_{Q} \approx 0.83 \mathrm{~km}$, next it has a nucleon-quark mixed phase layer with a thickness of $r_{M P} \approx 9.43 \mathrm{~km}$, followed by a normal nuclear matter layer with a thickness of $r_{N} \approx 0.45 \mathrm{~km}$.

\section{ACKNOWLEDGEMENTS}

The author would like to thank Prof. Yu. L. Vartanyan and Dr. G. S. Hajyan for fruitful discussions on issues related to the subject of this research. 


\section{REFERENCES}

[1] Serot, B.D. and Walecka, J.D. (1986) The relativistic nuclear many-body problem. In Adv. in Nuclear Physics, Eds., Negele, J.W and Vogt, E., 16(1), Plenum Press, New York.

[2] Liu, B., Greco, V., Baran, V., Colonna, M. and Di Toro, M. (2002) Asymmetric nuclear matter: The role of the isovector scalar channel. Physical Reviews $C$, 65(4), 335-345.

[3] Greco, V., Colonna, M., Toro, M.D. and Matera, F. (2003) Collective modes of asymmetric nuclear matter in quantum hadrodynamics. Physical Reviews C, 67(1), 015203.

[4] Glendenning, N.K. (1992) First-order phase transitions with more than one conserved charge: Consequences for neutron stars. Physical Reviews D, 46(3), 1274-1287,

[5] Heiselberg, H., Pethick, C.J. and Staubo, E.S. (1993)
Quark matter droplets in neutron stars. Physical Review Letters, 70(10), 1355-1359.

[6] Heiselberg, H. and Hjorth-Jensen, M. (2000) Phases of dense matter in neutron stars. Physics Reports, 328(5-6), 237-327.

[7] Alaverdyan, G.B. (2009) Relativistic mean-field theory equation of state of neutron star matter and a Maxwellian phase transition to strange quark matter. Astrophysics, 52(147), 132-150.

[8] Glendenning, N.K. (2000) Compact stars, Springer, Cambridge.

[9] Alaverdyan, G.B. (2009) Scalar-isovector $\delta$ meson in the relativistic mean field theory and the structure of neutron stars with a quark core. Gravitation \& Cosmology, 15(1), 5-9.

[10] Farhi, E. and Jaffe, R.L. (1984) Strange matter. Physical Reviews D, 30(2), 2379-2390. 